Provided for non-commercial research and education use. Not for reproduction, distribution or commercial use.

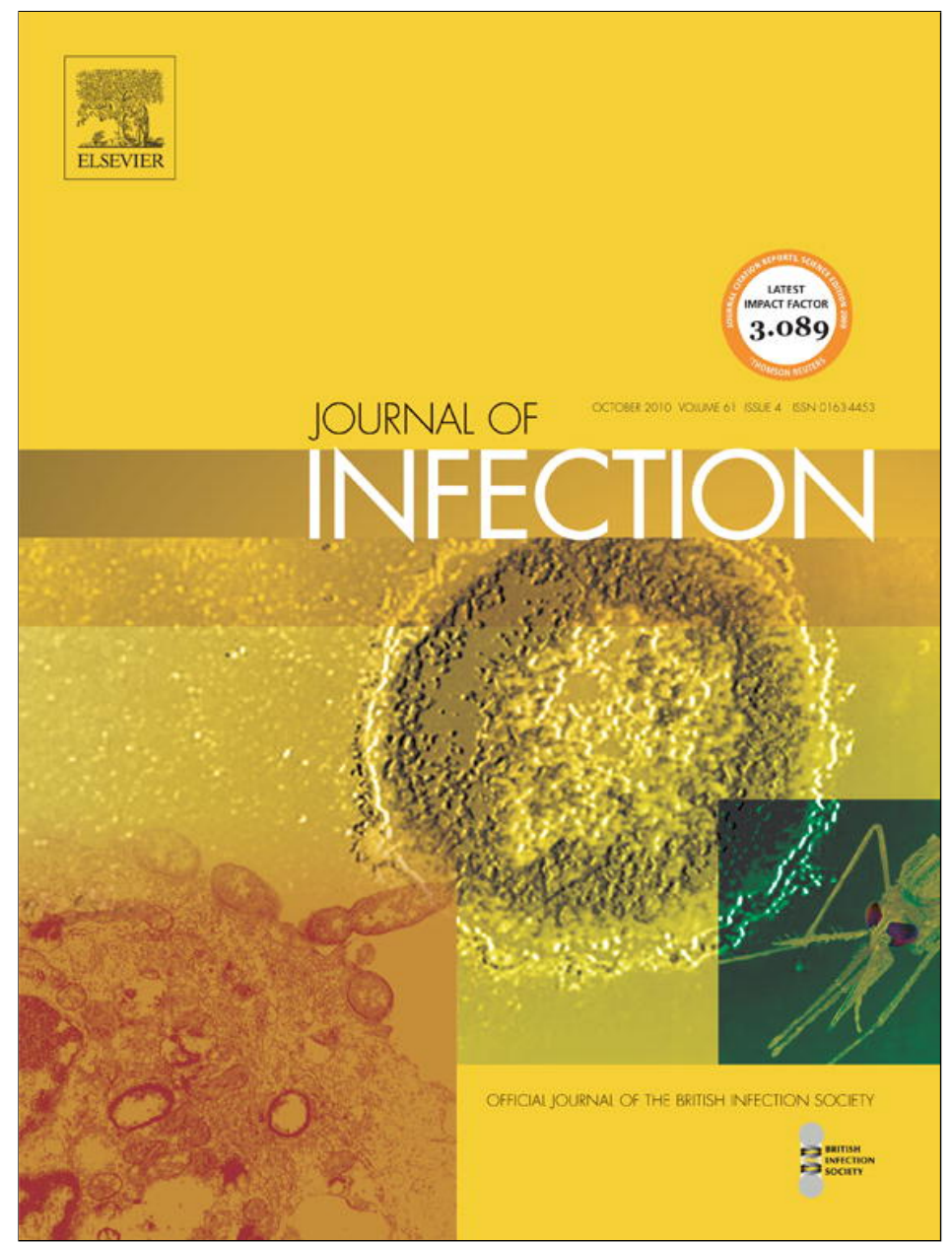

This article appeared in a journal published by Elsevier. The attached copy is furnished to the author for internal non-commercial research and education use, including for instruction at the authors institution and sharing with colleagues.

Other uses, including reproduction and distribution, or selling or licensing copies, or posting to personal, institutional or third party websites are prohibited.

In most cases authors are permitted to post their version of the article (e.g. in Word or Tex form) to their personal website or institutional repository. Authors requiring further information regarding Elsevier's archiving and manuscript policies are encouraged to visit:

http://www.elsevier.com/copyright 


\title{
Finite size effects in multilayered polymer systems: Development of PET lamellae under physical confinement
}

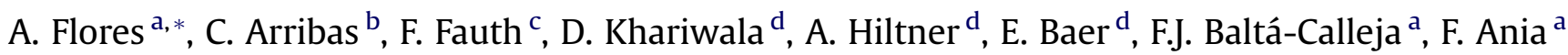 \\ ${ }^{a}$ Instituto de Estructura de la Materia, CSIC, Serrano 119, 28006 Madrid, Spain \\ ${ }^{\mathrm{b}}$ Dpto. Materiales y Producción Aeroespacial, E.T.S.I. Aeronáuticos, Universidad Politécnica de Madrid, Pza. Cardenal Cisneros 3, 28040 Madrid, Spain \\ ${ }^{c}$ BM16-CRG/Consorci Laboratori Llum Sincrotro (LLS) c/o European Synchrotron Radiation Facility (ESRF) BP220, F-38043 Grenoble, France \\ ${ }^{\mathrm{d}}$ Case Western Reserve University, 2100 Adelbert Road, Cleveland, OH 44106-7202, USA
}

\section{A R T I C L E I N F O}

\section{Article history:}

Received 17 March 2010

Received in revised form

29 July 2010

Accepted 3 August 2010

Available online 11 August 2010

\section{Keywords:}

Nanolayered PET

SAXS

Crystallization

\begin{abstract}
A B S T R A C T
The glass transition temperature and the crystallization behaviour of poly(ethylene terephthalate) PET ultra-thin layers (a few tens of $\mathrm{nm}$ ) within multilayered PET/polycarbonate (PC) coextruded films are investigated as a function of layer thickness by means of calorimetric measurements. Results are discussed in terms of reduced thickness and interface effects. The appearance and evolution of lamellar orientation upon isothermal crystallization of ultra-thin PET layers from the glassy state are explored based on real time small-angle X-ray scattering (SAXS) studies. Analysis of the SAXS measurements reveals that finite size effects hamper the crystallization process. However, the final lamellar structure is similar in both, the nanolayered PET and the bulk material. Results suggest that not only lamellar insertion but also some lamellar thickening contribute to the development of the final lamellar structure. Room temperature SAXS and wide-angle X-ray diffraction (WAXS) measurements indicate that two lamellar populations develop: edge-on lamellae are proposed to appear close to the interphases while flat-on lamellae, arising as a consequence of confinement, should be preferentially located in the layers core.
\end{abstract}

(c) 2010 Elsevier Ltd. All rights reserved.

\section{Introduction}

The current trends in Science and Technology concerning the development of nano-scaled materials require the understanding of fundamental phenomena taking place in confined media. In this respect, much effort has been carried out in Polymer Science in the last decade. Nanoconfinement can be achieved by means of chemical templates, such as amorphous blocks in crystallineamorphous block copolymers [1,2]. The self-assembly character of block copolymers can be exploited to produce a wide variety of topological nano-domains [2,3], including nanocylinders and nanolayers [4], nanoporous structures [5] and nanospheres [6]. In addition, other types of controlled molecular architecture, such as grafted polymers or hyperbranched polymers [7], can be used to generate different nanomaterials with great technological interest.

Alternatively, physical restrictions can also induce confinement effects by reducing at least one of the dimensions to nanometer scale. Thin films [8], multilayered materials [9] and intercalated

\footnotetext{
* Corresponding author.

E-mail address: imtf305@iem.cfmac.csic.es (A. Flores).
}

polymer-layered nanocomposites [10] belong to the class of nanoconfined materials in one direction (1-D), whereas nanorods [11] and nanofibres [12] represent examples of confined media in two directions. The former materials represent the simplest model to explore the effect of confined environments on the development of polymer morphology and nanostructure.

Studies of finite size effects on the glass transition temperature, $T_{\mathrm{g}}$, of polymer materials are especially profuse in case of thin films $[13,14]$. It appears that interface effects, related to both free and hard surfaces, determine to a great extent whether $T_{\mathrm{g}}$ increases, decreases or remains constant with film thickness [13,14]. Confinement effects are also found to influence the polymer dynamics $[15,16]$. It has also been suggested that the film creation process can have important consequences in the material behaviour [14]. Although studies of the relaxation behaviour of supported and free-standing thin polymer films offer diverse results, a broadening of the distribution of relaxation times is often reported as film thickness decreases $[14,17,18]$.

Finite size effects in multilayer films of different polymers have also been explored. The potential of these materials for technological applications rely on the adequate combination of the properties of the individual homopolymers. The behaviour of $T_{\mathrm{g}}$ with decreasing layer thickness has been examined in case of 
bilayer and trilayer films, fabricated from homopolymer layers deposited via spin-coating. It was shown that the $T_{\mathrm{g}}$ reduction due to enhanced mobility at the free surface can be tuned by changing the thickness and nature of the underlying polymer support [19]. Recently, the dynamics of multilayered films with tens to thousands of alternating layers of two immiscible polymers has been studied [20]. Films were prepared using a layer-multiplying coextrusion process that has been proved to produce highly regular structures $[9,21]$. Compared to multilayer polymers fabricated using spin-coating, layer-multiplying coextrusion can easily produce large amounts of material containing thousands of alternating layers, with individual thicknesses that can approach the nanoscale. Such forced-assembly films exhibit interphase regions that can have an important impact in the material properties. In case of nanolayered films, the interphase thickness can approach the scale of the individual nanolayers, hence, creating a new material that is entirely interphase [9]. Calorimetric studies on the $T_{\mathrm{g}}$ of layered nanostructures prepared by multiplying coextrusion reveal that the $T_{\mathrm{g}}$ of the individual polymers gradually shift closer together as layer thickness decreases, approaching a single value for layer thicknesses in the range of the interphase thickness [9]. Simultaneously, the width of the glass transition broadens considerably. Dynamic mechanical thermal analysis (DMTA) of various forced-assembly multilayered polymers confirm the occurrence of two $\tan \delta$ peaks for the film with discrete layers and a single broad peak for the interphase material [20].

Preceding studies show that multilayer films exhibit a crystallization behaviour different from the homopolymer counterparts [22], as it is also observed in case of thin films [23,24]. Reduced levels of crystallinity at the end of a delayed crystallization process are detected with increasing confinement for layer-multiplying coextrusion materials [25-27]. In addition, preferred lamellar crystal orientation seems to accompany the crystallization process under confinement. Polyethylene oxide (PEO) flat-on lamellae ( $c$-axis perpendicular to the surface layer) are found to emerge in layer-multiplying coextrusion films, for PEO layer thicknesses below $110 \mathrm{~nm}$ [22]. In contrast, edge-on lamellae (c-axis parallel to the surface layer) have been found in other nanolayered materials containing polyethylene (PE) or isotactic polypropylene (PP) as the crystallizable homopolymer $[27,28]$. In self-assembled multilayer block copolymers and thin films, there are experimental evidence that the crystallization temperature and/or thickness of the layers determine whether edge-on or flat-on lamellae should exist predominantly in the material $[1,23,29]$. It is generally observed that lower temperatures and thicker films favor edge-on morphologies $[23,29]$. Although some models have been recently proposed $[29,30]$, the mechanism that controls the specific lamellar orientation in confined media is still unclear.

In order to extend preceding studies concerning the crystallization behaviour of confined polymer films, layer-multiplying coextruded films of poly(ethylene terephthalate) (PET) and polycarbonate (PC) with layer thicknesses down to a few tens of nanometers are here investigated. Previous room temperature $\mathrm{X}$-ray diffraction measurements with the beam parallel to the PET/ PC layer stacking suggest that lamellar crystal orientation occurs for thin layer thicknesses below $300 \mathrm{~nm}$ [25]. In addition, isothermal crystallization experiments using a synchrotron radiation source reveal that the crystallization of PET within the multilayered materials is delayed with decreasing layer thickness [26]. These last measurements were carried out with the X-ray beam perpendicular to the film surface and anisotropic effects were not observed. The present paper offers a detailed study of the appearance and evolution of lamellar orientation in thin films of PET/PC multilayers, as revealed by real time small-angle X-ray diffraction measurements with the incident beam parallel to the layer stacking. We also report room temperature wide-angle X-ray diffraction studies that provide complementary information of chain orientation as a consequence of reduced thickness and interface effects. The influence of layer thickness in the glass transition temperature is also discussed.

\section{Experimental}

\subsection{Materials}

Poly(ethylene terephthalate) (PET) (Eastman PET9921) and polycarbonate (PC) (Bayer Makrolon 2207) were used in this work. Both materials have a molecular weight of about $30,000 \mathrm{~g} / \mathrm{mol}$. The coextrusion process used to prepare PET/PC 50/50 (vol/vol) microlayered and nanolayered materials was described previously [25], however, here a $36 \mathrm{~cm}$ exit die was used. The number of alternating layers was held constant at 1024 . The thickness of the films was varied in the range $20-214 \mu \mathrm{m}$ to produce different layer thicknesses. Extruded films of $\sim 25 \mathrm{~cm}$ wide (the precise width varied according to the layer thickness) and various meters long were obtained. The nominal PET and PC layer thicknesses for each sample varied in the range $20-210 \mathrm{~nm}$ (calculated from the number of layers and the film thickness).

\subsection{Differential scanning calorimetry (DSC) measurements}

A Perkin-Elmer DSC-7 differential scanning calorimeter was used to measure glass transition temperatures. The instrument was calibrated with indium. Samples of 6-8 $\mathrm{mg}$, extracted from the central part of the extruded sheets, were sealed in aluminum pans. All measurements were carried out at a heating rate of $20^{\circ} \mathrm{C} / \mathrm{min}$, under nitrogen atmosphere. The $T_{\mathrm{g}}$ values were determined as the midpoint of the heat capacity change.

A major concern in the determination of $T_{\mathrm{g}}$ in thin polymer films is the annealing history of the sample [13]. In this work, a thermal protocol was established to overcome the influence of thermal history, physical ageing and residual stresses on the chain conformations. Samples for calorimetric measurements were first introduced in a Mettler hot-stage at $85{ }^{\circ} \mathrm{C}$, kept at this temperature for $15 \mathrm{~min}$, and subsequently quenched in iced water. DSC scans were recorded immediately after the heat treatment. The annealing times applied to the amorphous PET samples at $85^{\circ} \mathrm{C}$ were chosen to be larger than the Rouse and retraction times reported for a PET sample of similar molecular weight $(2 \mathrm{~s}$ and $11 \mathrm{~min}$ respectively at $85^{\circ} \mathrm{C}$ ) [31]. Application of annealing times in the range of the reptation time (420 min) was discarded in order to ensure that the sample did not develop any trace of incipient crystallization. It should be noted that the glass transition temperature of the annealed material at $85{ }^{\circ} \mathrm{C}$ was found to be significantly different from that of the starting un-treated sample and remained constant in the second and third DSC heating runs (a rapid cooling after a few minutes above $T_{\mathrm{g}}$ was applied between scans).

The crystallization behaviour was explored in separate DSC heating runs where the temperature reached $260{ }^{\circ} \mathrm{C}$. The average value and error of the $T_{\mathrm{g}}$ and the crystallization temperature, $T_{\mathrm{c}}$, data were calculated from the measurement of at least two DSC scans of different samples from the same sheet. Data with no error bars correspond to a single value obtained from a unique DSC scan.

\subsection{Atomic force microscopy studies}

Atomic force microscopy (AFM) imaging was performed using the tapping mode of a Nanoscope IIIa (Digital Instruments). Phase and height images were recorded simultaneously. Specimens were sectioned perpendicular to the plane of the film with an 
ultramicrotome (MT6000-XL from RMC, Tucson, AZ, USA). For this purpose, a film specimen was embedded in epoxy and cured overnight at $23{ }^{\circ} \mathrm{C}$. Cured specimens were microtomed at $23^{\circ} \mathrm{C}$ at a cutting speed of $0.5 \mathrm{~mm} / \mathrm{s}$.

\subsection{Small-angle $X$-ray scattering}

\subsubsection{Isothermal crystallization experiments}

Real time small-angle X-ray scattering (SAXS) measurements of chosen PET/PC and PET amorphous samples were carried out at the BM16-CRG beamline at the ESRF, Grenoble, in order to follow the kinetics of lamellar crystallization at $150{ }^{\circ} \mathrm{C}$. The wavelength was $0.138 \mathrm{~nm}$ and the sample-detector distance was of $3492 \mathrm{~mm}$. The experimental set-up allowed resolving diffraction angles larger than $2 \theta=0.087^{\circ}$. A marCCD detector with $2048 \times 2048$ pixels was employed. Data were collected in binning mode resulting in $158.9 \mu \mathrm{m}$ effective pixel size. The intensity values were corrected for the background and normalized for the primary beam intensity taking into account the intensity of the ionization chamber located close to the beamstop. A Linkam hot-stage (model THMS600) was used with a sample holder that was especially designed to allow the positioning of the films with the beam parallel to the stacking of the layers (F or $\mathrm{T}$ direction according to Fig. 1 ).

\subsubsection{Lamellar orientation}

Weakly oriented SAXS patterns were obtained at the end of the crystallization process in PET/PC multilayered materials. As an example, Fig. 1 shows the pattern developed after the PET/PC 50/50 sample (63 nm PET layer thickness) was maintained at $150{ }^{\circ} \mathrm{C}$ for $70 \mathrm{~min}$. The strong meridional streak is associated with grazing incidence scattering arising from the interfaces between the two homopolymers, probably due to the presence of nanogaps, and
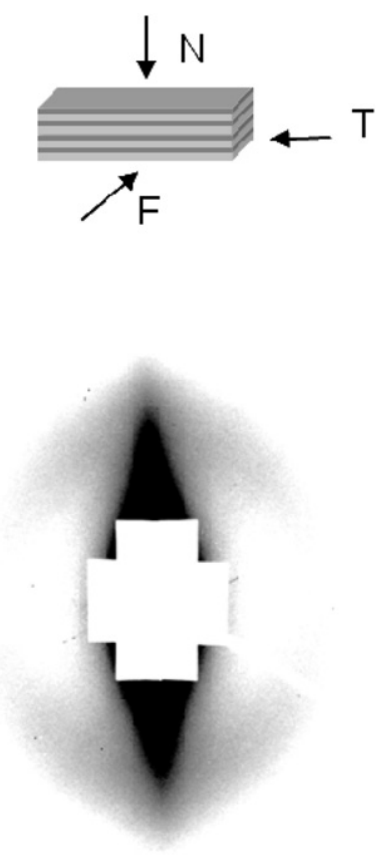

F or T dircction

Fig. 1. SAXS pattern of PET/PC 50/50 with $63 \mathrm{~nm}$ layer thickness after 70 min at $150{ }^{\circ} \mathrm{C}$ The film thickness was positioned with the layer stacking parallel to the incident beam ( $F$ or $T$ directions). A large beamstop was placed to avoid damage of the detector due to the intense meridional streak arising from the outer surfaces and the interfaces between the two homopolymers. from the outer surfaces. In order not to saturate the detector, the intensity of this meridional streak was reduced by tilting the samples a few degrees away $\left(\sim 5^{\circ}\right)$ from the plane defined by the cross-section of the incident beam; additionally, a large beamstop was placed. In this way, although the intensity of the meridional streak is still high as shown in Fig. 1, the appearance of a diffraction maximum with slight anisotropy can be detected. In order to follow the changes in the lamellar stack orientation during the crystallization process, the azimuthal scan of each image at a radius corresponding to the maximum of intensity was obtained as shown in Fig. 2 and analyzed as follows. Firstly, we have used least-squares analysis to separate the contribution of the background (horizontal line in Fig. 2) from the meridional streak (described by a Lorentzian function) and the distribution of orientations arising from the lamellae (described by a Gaussian). Room temperature SAXS measurements taken before the isothermal crystallization experiments reveal that the corresponding azimuthal profile can be fitted to Lorentzian functions with no contribution of Gaussian peaks. Next, the orientation function was calculated following:

$f=\frac{3\left\langle\cos ^{2} \varphi\right\rangle-1}{2}$

where $\varphi$ is the azimuthal angle. It should be noted that the orientation function provides information on the distribution of orientations along a preferential direction and not on the total amount of oriented material.

\subsubsection{Lamellar nanostructure}

To extract information on the lamellar nanostructure, a detailed analysis of the SAXS curves was carried out. SAXS patterns were azimuthally integrated with the help of FIT2D software package to obtain a curve of intensity, $I$, as a function of the wave vector, $q(q=4 \pi \sin \theta / \lambda)$ [32]. In weakly oriented systems, application of the azimuthal averaging algorithm is permitted [33]. As an example, Fig. 3 illustrates the $I(q)$ curve for the PET/PC material with PET layer thickness of $63 \mathrm{~nm}$ after $7 \mathrm{~min}$ at $150{ }^{\circ} \mathrm{C}$. The limited $q$ range available did not allow for a proper analysis of the SAXS data by means of the correlation or interface distribution functions [33]. Indeed, a linear Porod region was not observed because sufficiently large $q$ values were not recorded. Alternatively, we have analyzed our data using the intensity profile method described in Refs. $[34,35]$. It has been shown that this procedure yields similar results than conventional correlation function analysis. However, the intensity profile method presents the advantage of univocally determining the thickness of the crystal and the amorphous layers, as well as the possibility of estimating the coherence length of the lamellar stacks.

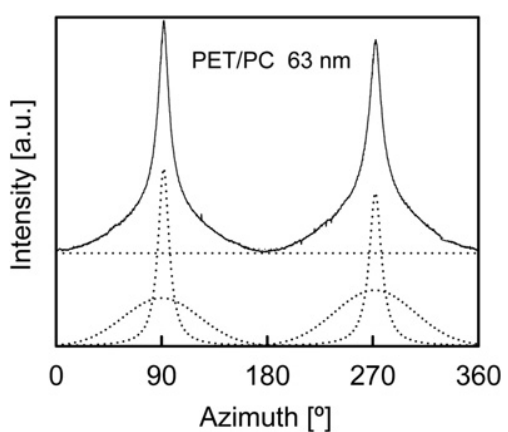

Fig. 2. Azimuthal SAXS profile of the PET/PC 50/50 $63 \mathrm{~nm}$ material, after $38 \mathrm{~min}$ at $150{ }^{\circ} \mathrm{C}$. A section of 20 pixels around the maximum of intensity was used for each azimuthal angle. The separate contributions of the background (horizontal line), the meridional streak (Lorentzian function) and the distribution of orientations arising from the lamella (Gaussian function) can be clearly distinguished. 


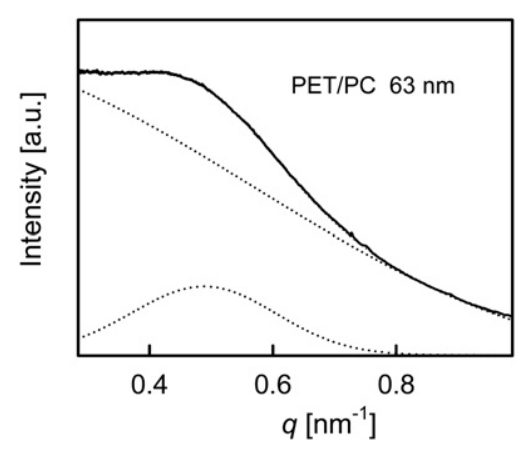

Fig. 3. Intensity curve as a function of wave vector for the PET/PC material with layer thickness of $63 \mathrm{~nm}$ after $7 \mathrm{~min}$ at $150{ }^{\circ} \mathrm{C}$. The curve was obtained from the azimuthal integration of the corresponding 2-D pattern using FIT2D software. The dotted lines illustrate the $I_{\mathrm{D}}$ and $I_{\mathrm{L}}$ and contributions to the total diffracted intensity, as shown in equation $(2)\left(I_{\mathrm{B}}=0\right)$.

In the intensity profile analysis, the observed intensity is described as

$I=I_{\mathrm{B}}+I_{\mathrm{D}}+I_{\mathrm{L}}$

where $I_{\mathrm{B}}$ is the background intensity which is assumed to be linear, $I_{\mathrm{D}}$ represents the diffuse scattering and $I_{\mathrm{L}}$ accounts for the lamellar peak. $I_{D}$ can be expressed as

$I_{\mathrm{D}}=\left[a_{1} F_{\text {step }}\left(a_{2}\right)\right]^{2}$

$F_{\text {step }}\left(a_{2}\right)=\sin \left(a_{2} q\right) /\left(a_{2} q\right)$

Furthermore, $I_{\mathrm{L}}$ can be written as

$I_{\mathrm{L}}(q)=\left[F_{\text {step }}\left(a_{3}\right)\left\{\operatorname{Gauss}\left(a_{4}, a_{5}, a_{6}\right)+\operatorname{Gauss}\left(a_{4}, 2 a_{5}, a_{6}\right)\right\}\right]^{2} / q^{2}$

where Gauss $\left(a_{4}, a_{5}, a_{6}\right)=a_{4} \exp \left[-0.5\left(\left(q-a_{5}\right) / a_{6}\right)^{2}\right], q^{2}$ is the Lorentz factor; $F_{\text {step }}\left(a_{3}\right)$ is a function analogous to the one shown in equation (3) and represents the structure factor of the lamellae; Gauss $\left(a_{4}, a_{5}, a_{6}\right)$ and $\operatorname{Gauss}\left(a_{4}, 2 a_{5}, a_{6}\right)$ are the first and second-order interference functions respectively.

The experimental intensity curves are fitted to equation (2) and the parameters $a_{1}, a_{2}, a_{3}, a_{4}, a_{5}, a_{6}$ are derived. As an example, Fig. 3 illustrates the $I_{\mathrm{D}}$ and $I_{\mathrm{L}}$ functions fitted to the intensity curve; we have set $I_{\mathrm{B}}=0$ through the whole series of experiments as it has been shown to produce more consistent results. However, the uncertainty in the derivation of the $a_{1}-a_{6}$ parameters is relatively large partly due to the limited angular range available. To reduce this error, we adopted the following procedure. First, a plot of $I q^{2}$ versus $q$ was fitted to a Pearson IV function using least-squares analysis. The position of the maximum of this function, $q_{\max } \pm \sigma$, was used as a first approximation to the lamellar spacing value, $L\left(L=2 \pi / q_{\max }\right)$. Next, $q_{\max }$ was used to set an initial value for the $a_{5}$ parameter ( $a_{5}=q_{\mathrm{max}}$, see equation (7) below) that was allowed to vary in the range $q_{\max } \pm \sigma$. Following this procedure, the standard error for each $a_{\mathrm{n}}$ parameter was limited to less than $12 \%$. Finally, the structural parameters of the lamellae are given by

$l_{\mathrm{a}}=2 a_{3}$

$L=2 \pi / a_{5}$

$L_{\mathrm{coh}}=4 \pi /\left[a_{6} \sqrt{(8 \ln 2)}\right]$ where $l_{\mathrm{a}}$ is the thickness of the amorphous layers in the lamellar stacks and $L_{\text {coh }}$ the coherence length of the lamellar stacks. The crystal thickness, $l_{\mathrm{c}}$, is calculated according to: $l_{\mathrm{c}}=L-l_{\mathrm{a}}$.

\subsection{Wide-angle X-ray scattering}

The crystal orientation and crystal structure upon crystallization were investigated by means of wide-angle X-ray scattering (WAXS) measurements. Room temperature two-dimensional patterns of the crystallized materials were obtained using a Micro Star rotating anode generator with copper target manufactured by Bruker (Germany). WAXS patterns were recorded using a Mar345 dtb image plate with a resolution of $3450 \times 3450$ pixels and $100 \mu \mathrm{m} /$ pixel; a sample-to-detector distance of $200 \mathrm{~mm}$ was used. The patterns were analyzed using FIT2D. In order to determine the crystal orientation, the CLEARER2 software package was employed [36]. Fibre WAXS patterns can be simulated from user-input atomic coordinates and unit cell parameters. The flexibility of CLEARER2, allowing any orientation of the fibre axis and of the incident beam, is most valuable for the determination of the axis of symmetry in uniaxial systems, as will be shown below.

\section{Results and discussion}

\subsection{Influence of layer thickness on the glass transition temperature}

Fig. 4 illustrates the variation of the $T_{\mathrm{g}}$ of PET, determined by means of DSC, as a function of nominal layer thickness for the multilayered materials. The $T_{\mathrm{g}}$ value for the PET homopolymer is also plotted as 'non-confined'. The $T_{\mathrm{g}}$ values of PC within the PET/PC multilayered materials could not be determined by means of DSC because the glass transition takes place at temperatures where the crystallization of PET already starts $\left(T_{\mathrm{g}}\right.$ of $\left.\mathrm{PC} \approx 150{ }^{\circ} \mathrm{C}\right)$. Results show a clear $T_{\mathrm{g}}$ increase for all the multilayered samples with respect to the PET homopolymer. This upwards $T_{\mathrm{g}}$ shift could be explained as due to, at least, one of the two following reasons: i) the diffusion of PC molecules into the PET layers: previous work in multilayered glassy polymers explained the progressive convergence of the $T_{\mathrm{g}}$ of the two homopolymers to a single value observed for ultra-thin layers as due to the diffusion of molecules of one polymer pair into the other [9]; ii) hindered molecular mobility due to the interaction between the PET molecules and the interphases. In supported thin polymer films, the polymer-substrate interaction often retards chain mobility due to an increased mass density at the interface [29]; similarly, in multilayered materials, the polymer-interface interaction could lead to an enhancement of $T_{\mathrm{g}}$. At present we are unable to discern the predominant mechanism responsible for the observed $T_{\mathrm{g}}$ shift. It is noteworthy that

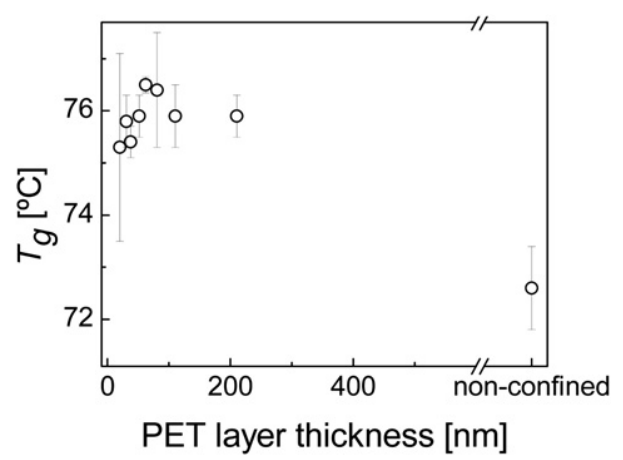

Fig. 4. Plot of $T_{\mathrm{g}}$ as a function of nominal layer thickness for the PET/PC multilayered series. The $T_{\mathrm{g}}$ value for the PET control material is included as 'non-confined'. 
preceding published work on freely standing films suggests that chain confinement effects turn out in a $T_{\mathrm{g}}$ decrease with decreasing thickness [37]. Such decrease is not clearly observed in our multilayered PET/PC series (see Fig. 4), hence, chain confinement does not seem to play a predominant role in the material average molecular dynamics for the range of layer thicknesses investigated.

\subsection{Changes in the crystallization behaviour by means of calorimetric data}

Fig. 5 (top) illustrates the values of the crystallization temperature, $T_{\mathrm{C}}$, of PET determined from DSC measurements, as a function of nominal layer thickness within the PET/PC 50/50 multilayered materials. Starting from the non-confined value, a significant $T_{\mathrm{c}}$ increase is observed when reducing the size of the layers down to a layer thickness of $63 \mathrm{~nm}$. However, for layer thicknesses of $50 \mathrm{~nm}$ and below, the $T_{\mathrm{c}}$ values are again comparable to that found for the non-confined material. Fig. 5 (bottom) illustrates representative DSC scans for the multilayered samples with layer thicknesses in the range $20-80 \mathrm{~nm}$. It is seen that samples with PET layer thicknesses of 20,30, 37 and $50 \mathrm{~nm}$ exhibit a well-defined and narrow crystallization peak in the DSC scan, similar to that observed for the PET homopolymer. In contrast, the materials with layer thicknesses of 63 and $80 \mathrm{~nm}$ display a broad crystallization peak shifted towards higher temperatures $\left(T_{\mathrm{c}} \approx 173^{\circ} \mathrm{C}\right)$. It is noteworthy that the same crystallization behaviour was found for both, the freshly quenched samples and the aged materials. This result is in agreement with preceding studies revealing that polymer ultra-thin layers exhibit a broad relaxation distribution as compared to the bulk $[17,38,39]$. It seems that the finite size effects effectively hamper the crystallization behaviour of PET for layer thicknesses of 63 and $80 \mathrm{~nm}$. What could be the reason for the sudden drop of the crystallization temperatures below $63 \mathrm{~nm}$ ? Fig. 6 illustrates the AFM images of the $80 \mathrm{~nm}$ and the $30 \mathrm{~nm}$ layered material, i.e., above and below the threshold for the change observed in crystallization behaviour. A layered structure is observed for both
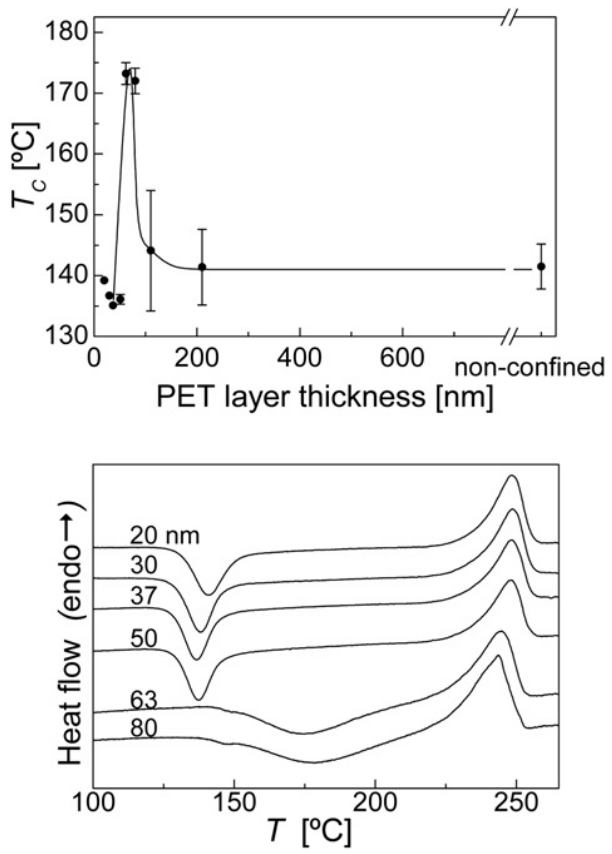

Fig. 5. (Top) Plot of $T_{\mathrm{c}}$ as a function of nominal layer thickness for the PET/PC series (Bottom) Representative DSC scans of the PET/PC multilayered materials with layer thicknesses indicated in the figure $(20-80 \mathrm{~nm})$. materials, however the $30 \mathrm{~nm}$ one exhibits layer instability; adjacent layers even seem to nearly connect at certain locations. This layer waving could be further deteriorated upon heating above the glass transition temperature of PET resulting in regions where finite size effects are limited and the crystallization behaviour would approach that of bulk PET. In addition, one cannot ignore the fact that as the layer thickness decreases approaching the radius of gyration of PET (of $4-9 \mathrm{~nm}[40,41]$ ), chain confinement effects could play a significant role in the crystallization behaviour, leading to a reduction in the $T_{\mathrm{c}}$ values.

\subsection{Real time SAXS experiments}

Fig. 7 illustrates the azimuthally integrated SAXS patterns of selected layered structures, recorded in-situ at $150^{\circ} \mathrm{C}$, as a function of crystallization time (from left to right): PET, PET/PC with $63 \mathrm{~nm}$ layer thickness and PET/PC with $37 \mathrm{~nm}$ layer thickness. It is clearly seen that crystallization starts at a later stage in the sample with $63 \mathrm{~nm}$ layer thickness, in agreement with the delayed crystallization process found for this material by means of DSC. In addition, no significant difference is found between the induction period for PET and that for the $37 \mathrm{~nm}$ thickness material, within the resolution of the experiment (the accumulation time was set to 29 and $59 \mathrm{~s}$ respectively).

\subsubsection{Analysis of the lamellar structure during crystallization}

In order to further understand the effect of finite size in the development of the lamellar structure, we have next analyzed the SAXS crystallization experiments of the PET/PC material with layer thickness of $63 \mathrm{~nm}$ and the PET control sample by means of the intensity profile analysis described in the Experimental section. Fig. 8 illustrates the lamellar long period, crystal thickness and amorphous thickness as a function of crystallization time at $150{ }^{\circ} \mathrm{C}$ for both materials. For the sake of clarity, error bars are only included for the PET/PC sample. It can be observed that, in both cases, the long period decreases as isothermal crystallization proceeds. A similar behaviour has been previously observed in PET crystallized from the glassy state and from the melt [42-45]. This experimental fact has been a subject of controversy for the last years, especially because the criterion used in the SAXS analysis to attribute one layer thickness, or the other, to the lamellar crystals has given rise to different interpretations in the development of the lamellar structure upon isothermal crystallization [42-46]. In case of melt crystallized material, crystal and amorphous thicknesses are found to decrease or remain constant as crystallization proceeds $[44,45]$. These observations are usually explained as arising from the progressive insertion of lamellae between the existing ones, or due to changes in the distribution and number of the amorphous layers. In contrast, cold crystallization studies suggest that $l_{\mathrm{a}}$ decreases and $l_{c}$ remains constant or slightly increases upon crystallization, although the precise mechanism associated has not been discussed in detail $[26,43]$. The results shown in Fig. 8 for the PET control material are in agreement with preceding experiments. However, most interesting is the fact that for the layered material the crystallization process is delayed; here, the crystal thickness values clearly increase in the first stages of crystallization, while the amorphous layer thickness decreases. This result suggests that the crystallization process cannot be simply explained by a lamellar insertion model (supported by the fact that the $L$ values decrease with increasing crystallization time) but some crystal thickening should also take place. The discussion is now opened as to whether the initial $l_{\mathrm{c}}$-rise observed for the layered material is not detected in case of the PET control due to the time scale of the experiment. Indeed, recent SAXS experiments on the same PET control sample, with the incident beam perpendicular to 


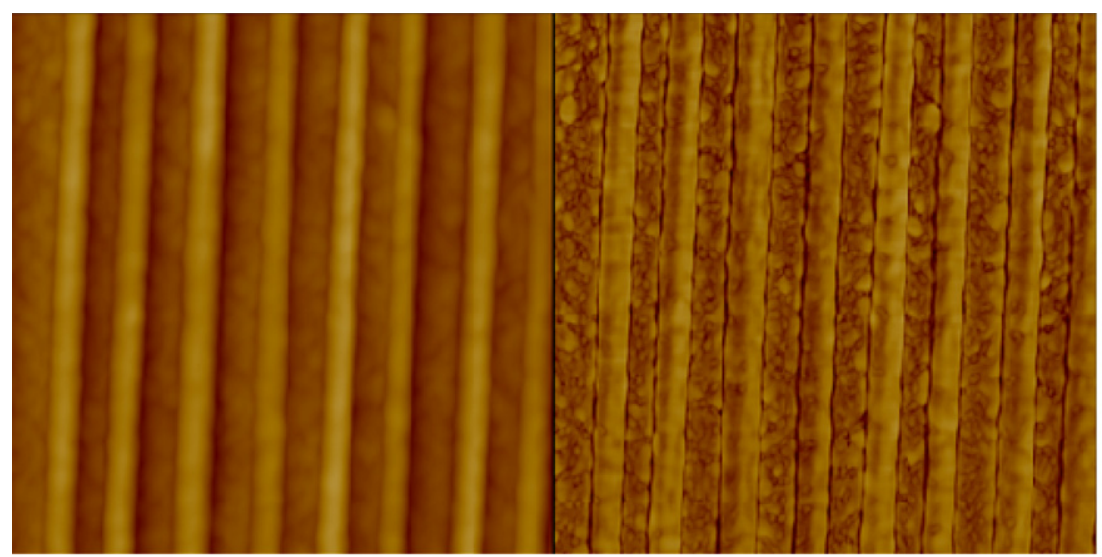

0

$1.25 \mu \mathrm{m}$

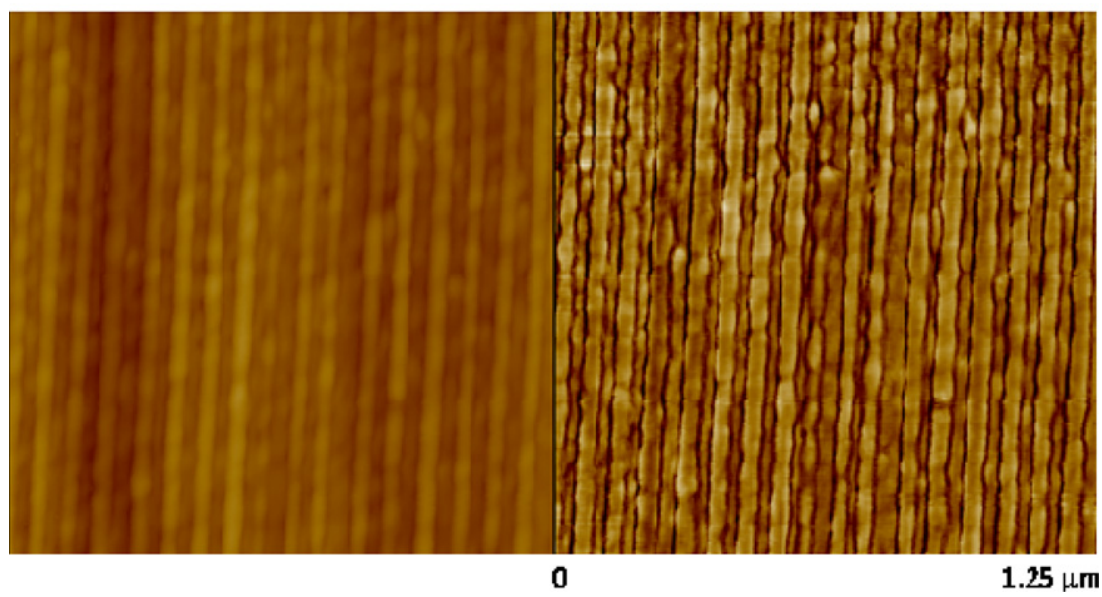

Fig. 6. Height (left) and phase (right) atomic force microscopy images of: (top) $80 \mathrm{~nm}$ and (bottom) $30 \mathrm{~nm}$ layered PET/PC samples.

the surface layers, carried out at a lower crystallization temperature of $117^{\circ} \mathrm{C}$, suggest that crystal thickness effectively increases during the early stages of crystallization.

The results of Fig. 8 also show that the multilayered material exhibits larger long period values at all stages of the crystallization process, compared to those of the PET control sample. This is a consequence of the larger amorphous layers between crystal lamellae. It is noteworthy that both $l_{\mathrm{c}}$ and $l_{\mathrm{a}}$ of the layered material differ significantly from those of the PET control sample in the first stages of crystallization while for long crystallization times only

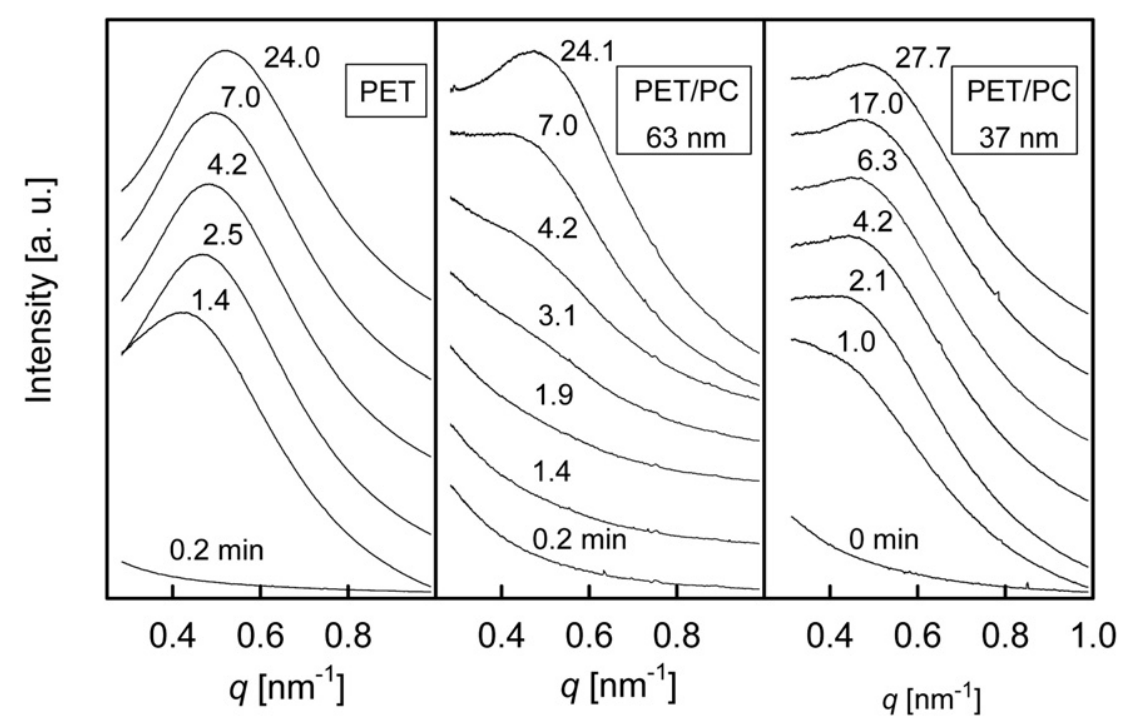

Fig. 7. Azimuthally integrated SAXS patterns versus $q$ recorded at various crystallization times during an isothermal crystallization experiment at $150{ }^{\circ} \mathrm{C}$ for (from left to right): PET, $\mathrm{PET} / \mathrm{PC}$ with $63 \mathrm{~nm}$ layer thickness and PET/PC with $37 \mathrm{~nm}$ layer thickness. The crystallization time corresponding to each intensity curve is included in the figure. Curves are shifted for the sake of clarity. 


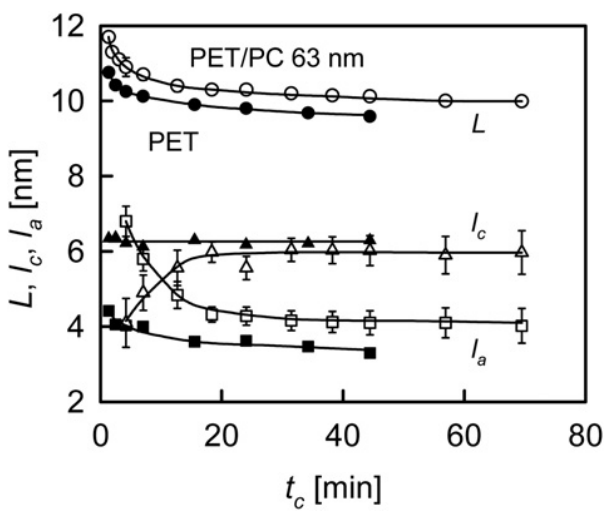

Fig. 8. Lamellar long period $(\bullet, \bigcirc)$, crystal thickness $(\boldsymbol{\Delta}, \Delta)$ and amorphous thickness $(\boldsymbol{\square}, \square)$ as a function of crystallization time at $150{ }^{\circ} \mathrm{C}$ for PET (solid symbols) and the PET/PC sample with $63 \mathrm{~nm}$ layer thickness (open symbols).

a moderate difference in the $l_{\mathrm{a}}$ values of both materials is observed. In other words, finite size effects delay the crystallization process but have little effect in the final lamellar structure, a result that has been previously suggested [26]. It should be noted that PET lamellar crystals are usually constituted by only a few monomer units, typically $3-4$. Hence, thinner crystals are probably unstable for thermodynamic considerations.

It is noteworthy that the $L, l_{\mathrm{c}}$ and $l_{\mathrm{a}}$ values for the PET control sample at the end of the crystallization process are in good agreement with preceding data for cold crystallized PET at $150^{\circ} \mathrm{C}$, which were analyzed using the correlation distribution function [46]. It should also be mentioned that, based on the intensity profile analysis of the SAXS curves, lamellar coherence length values are found to be $\sim 30 \mathrm{~nm}$ suggesting that an average of $\sim 3$ lamellar crystals constitute the lamellar stacks. Finally, we have found that the lamellar coherence length values exhibit a tendency to increase with increasing crystallization time, in accordance with lamellar insertion as a relevant mechanism during the crystallization process.

\subsubsection{Development of lamellar orientation}

Fig. 9 shows the orientation distribution function obtained for the PET lamellar crystals within the $63 \mathrm{~nm}$ layers in the course of the isothermal crystallization process at $150{ }^{\circ} \mathrm{C}$. It is seen that the lamellar orientation appears at the early stages of crystallization, immediately after reaching $150{ }^{\circ} \mathrm{C}$ and decreases afterwards as crystallization proceeds. It is noteworthy that the changes in the orientation function during crystallization coincide with the

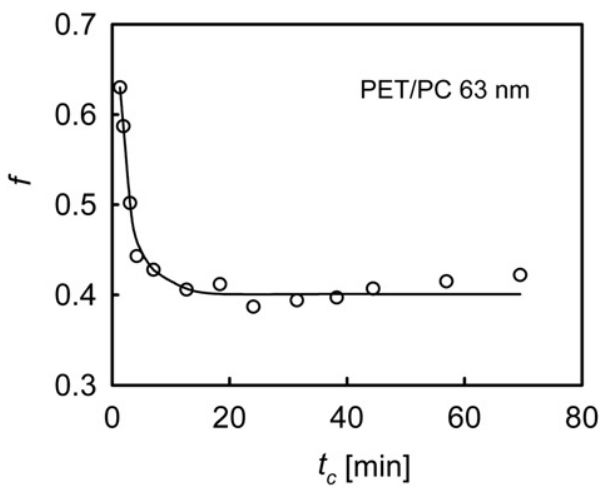

Fig. 9. Orientation distribution function versus crystallization time at $150{ }^{\circ} \mathrm{C}$. A guide-line is shown. changes in the structural parameters (see Fig. 8), both levelling-off after $\sim 20$ min of crystallization time. The broadening of the orientation distribution function in the first stages of the crystallization process, reflected as a decrease of the $f$-parameter in Fig. 9, should be associated to the appearance of new lamellae with the direction normal to the basal surface slightly away from the $\mathrm{N}$-direction of Fig. 1. We wish to remark that an inclination of the lamellar surface is frequently observed in case of cold-drawn PET, where the parallelization of the neighbouring molecules prior to crystallization seems to be associated with the consequent lamellar inclination [47]. In the $63 \mathrm{~nm}$ layered material, lamellar crystals develop in the first stages of crystallization with their surfaces being preferentially aligned perpendicular to the $\mathrm{N}$-direction, as suggested by the broad maximum centred on the meridian (see Fig. 1 as an example, see also the azimuthal scan of Fig. 2). After the development of the first crystals, the interlamellar amorphous regions retain some residual stress that could lead to inclined lamellae upon further crystallization. Fig. 10 schematically depicts this idea. Here, insertion of new lamellae with their basal surfaces slightly away from the plane defined by the layer stacking could explain the changes observed in the orientation distribution function.

\subsection{Room temperature SAXS studies}

After the isothermal crystallization process, room temperature SAXS measurements taken in the $\mathrm{F}$ and $\mathrm{T}$ directions revealed weakly anisotropic patterns, as shown in Fig. 11 (left). In contrast, an isotropic broad ring was observed when the film was positioned perpendicularly to the $\mathrm{X}$-ray beam ( $\mathrm{N}$-direction). The SAXS pattern of Fig. 11 resembles those obtained in the same sample at the end of the crystallization process (see for example Fig. 1). Similarly, the maximum intensity is concentrated on the meridian suggesting that lamellar surfaces are preferentially aligned parallel to the layer stacking (flat-on lamellae). Analysis of the corresponding orientation distribution function yields $f=0.49$, a value slightly larger than the plateau value shown in Fig. 9. In addition, the SAXS pattern of Fig. 11 (left) was azimuthally integrated and further analyzed by means of the intensity profile method yielding $L=9.7 \mathrm{~nm}$, $l_{\mathrm{a}}=4.5 \mathrm{~nm}$ and $l_{\mathrm{c}}=5.2 \mathrm{~nm}$. Comparison of these values with those shown in Fig. 8 for long crystallization times $(L=10.0 \pm 0.1 \mathrm{~nm}$; $l_{\mathrm{a}}=4.0 \pm 0.4 \mathrm{~nm}, l_{\mathrm{c}}=6.0 \pm 0.6 \mathrm{~nm}$ ) suggests that slightly thinner crystals (unstable at higher temperatures) separated by larger amorphous regions are generated during the cooling process to room temperature. Results also suggest that these thinner crystals

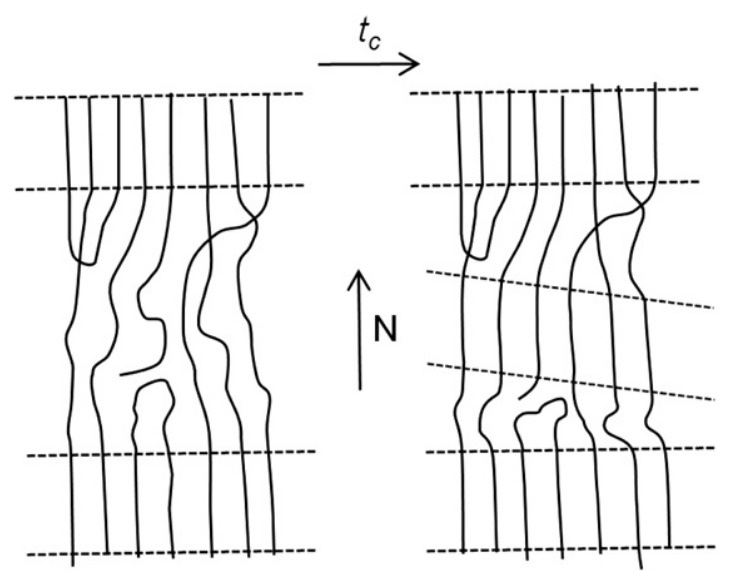

Fig. 10. Schematic representation of the insertion of new lamellae. Note that the perpendicular to the basal surfaces is slightly inclined with respect to the N-direction. 

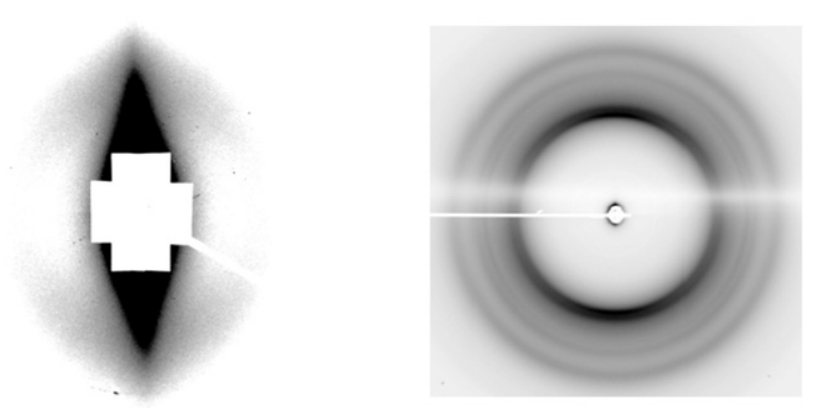

Fig. 11. Room temperature SAXS (left) and WAXS (right) patterns of the $63 \mathrm{~nm}$ PET/PC multilayered material crystallized at $150{ }^{\circ} \mathrm{C}$. The incident beam was directed parallel to the layer stacking.

exhibit an enhanced orientation with respect to those developed during the isothermal crystallization process. This is possibly due to the fact that the lamellar crystals generated at $150{ }^{\circ} \mathrm{C}$ induce an additional confinement effect on the development of the thinner crystals arising upon cooling leading to an enhanced average orientation.

\subsection{Development of crystal orientation as revealed by WAXS at room temperature}

Fig. 11 (right) illustrates the room temperature WAXS pattern along the $\mathrm{T}$ direction of the $63 \mathrm{~nm}$ sample after the isothermal crystallization experiment at $150{ }^{\circ} \mathrm{C}$. This diffraction pattern was taken on the same sample as that used for the SAXS pattern of Fig. 11 (left). Diffraction patterns in the F direction yield similar WAXS results. In contrast, continuous diffraction rings were found when the beam was directed perpendicularly to the surface of the film (N-direction). The WAXS pattern of Fig. 11 indicates preferential orientation of the lamellar crystals. On the other hand, the SAXS pattern on the left-hand side of the same figure suggests the appearance of flat-on lamellae, as discussed in the preceding section. Hence, one would expect a WAXS pattern in agreement to those well-known for uniaxially oriented PET [48], with the c-axis parallel to the vertical direction (N-direction) or with a small tilting angle. Strikingly, the WAXS pattern of Fig. 11 has no similarity with those of uniaxially oriented PET, in fact, it is indeed very similar to the patterns associated to lamellae having edge-on orientation $[49,50]$. The Bragg spacings associated to the main $(h k l)$ reflections were determined and agree well with the unit cell published by Bunn, as shown in Table 1 [51]. Aiming at acquiring a detailed knowledge of the precise crystal orientation of the edge-on lamellae, we have used the CLEARER2 program to simulate uniaxial diffraction patterns with different axis of symmetry. The simulated pattern is found to approach the experimental one when the axis of symmetry is a few degrees away from the $a-b$ plane of the unit cell, more precisely, when the $(i, j, k)$ coordinates of the axis of symmetry (with reference to the unit cell) take values of: $i \approx 0.4$, $j \approx 0.7$ and $k \approx 0.1$. As an example, Fig. 12 illustrates the WAXS pattern simulated using a fibre axis of $(0.37,0.73,0.09)$. The orientation of the unit cell with respect to the axis of symmetry is schematically depicted in the figure. It should be noted that the $c$-axis lays in the plane of the layer stacks, characteristic of edge-on lamellae.

\subsection{Coexistence of two lamellar populations}

WAXS results suggest the presence of edge-on lamellae, with the $c$-axis randomly oriented in the plane of the layers. This finding is in agreement with the occurrence of flattened spherulites,
Table 1

Experimental values of the $(h k l)$ plane spacings, $d_{\text {exp }}$, and calculated ones according to the PET unit cell published by Bunn, $d_{\text {Bunn }}[51]$.

\begin{tabular}{lll}
\hline Plane $(h k l)$ & $d_{\exp }[\mathrm{nm}]$ & $d_{\text {Bunn }}[\mathrm{nm}]$ \\
\hline$(0 \overline{1} 1)$ & 0.534 & 0.540 \\
$(010)$ & 0.513 & 0.506 \\
$(\overline{1} 11)$ & 0.413 & 0.417 \\
$(\overline{1} 10)$ & 0.394 & 0.394 \\
$(100)$ & 0.344 & 0.347 \\
\hline
\end{tabular}

schematically depicted in Fig. 13 (top), similar to those suggested for PET thin films [50]. Such lamellar arrangement would explain the isotropic SAXS pattern found when the beam is applied in the $\mathrm{N}$-direction. However, it cannot substantiate the preferential SAXS orientation found when the beam was directed parallel to the F and T directions (see Fig. 1 and left-hand side of Fig. 11). In this case, the maximum of intensity found in the meridian suggests the presence of flat-on lamellae, as mentioned in the preceding sections. Hence, we envisage the presence of two different lamellar populations:

- Edge-on lamellae give rise to well-defined diffraction arcs in the WAXS pattern of the multilayered film when the beam is directed along $\mathrm{F}$ or $\mathrm{T}$ (see Fig. 11, right). Isotropic WAXS and SAXS rings are expected for this lamellar orientation when the film surface is perpendicular to the incident beam, in agreement with the experimental results. It is noteworthy that the SAXS patterns obtained in the $\mathrm{F}$ and $\mathrm{T}$ directions do not show equatorial maxima (see Figs. 1, 2 and 11 left). The twisting, bending and small number of edge-on lamellae possibly preclude any SAXS orientation along this direction.

- Flat-on lamellae are associated to the meridional maxima observed in the SAXS patterns along the F and T directions (see Fig. 11 left). Such lamellar arrangement would not contribute to the SAXS pattern in the N-direction. On the other hand, there is no indication of flat-on lamellar orientation by means of WAXS. This is possibly due to the short lateral coherence length and the imperfect lamellar crystals produced under confinement effects.

Fig. 13 (bottom) schematically depicts a lamellar arrangement in agreement with our SAXS and WAXS results. In this scheme, edgeon lamellae are proposed to appear close to the interfaces, while flat-on lamellae should grow in the layer cores. It is suggested that flat-on lamellae are generated in the central part of the layers because preliminary SAXS studies in the PET/PC layered materials with different layer thicknesses (results not shown here) indicate
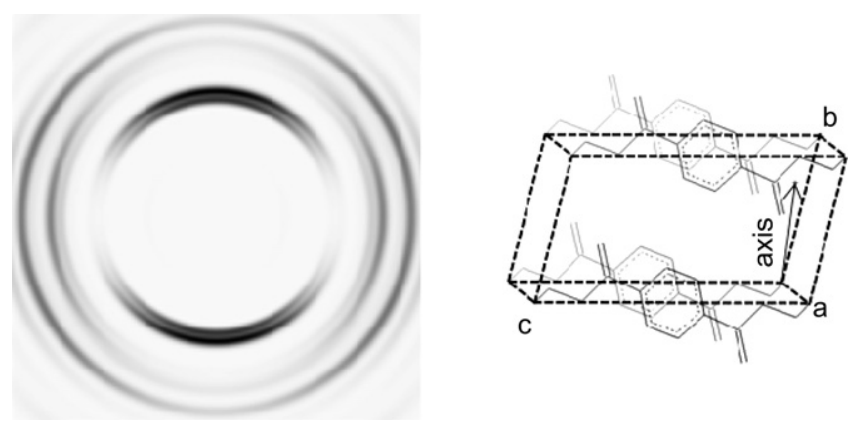

Fig. 12. (Left) Simulated room temperature WAXS pattern of the $63 \mathrm{~nm}$ PET/PC sample crystallized at $150{ }^{\circ} \mathrm{C}$. The predicted pattern was simulated with the help of the CLEARER2 program, using a uniaxial symmetry axis of $(0.37,0.73,0.09)$ as depicted in the right-hand side of the figure; the $(i, j, k)$ coordinates are referred to the PET unit cell published by Bunn [51]. 

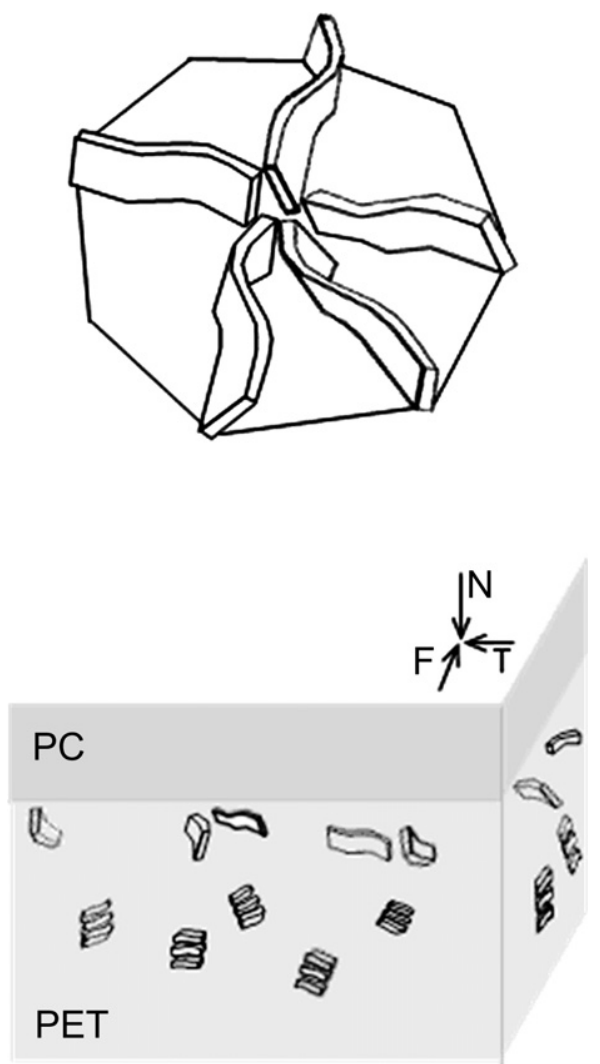

Fig. 13. Schematical representation of: (top) flattened spherulites, similar to those suggested for PET thin films [50]; (bottom) a proposed lamellar arrangement in agreement with SAXS and WAXS results: edge-on lamellae appear close to the interfaces while flat-on lamellae grow in the layer cores.

that the distribution of orientations along the $\mathrm{N}$-direction substantially narrows as the layer thickness decreases. A result that cannot be explained if flat-on lamellae were generated at the interfaces.

\section{Conclusions}

Finite size effects are explored in ultra-thin layers of PET within PET/PC multilayered films. Calorimetric measurements reveal that the glass transition temperature of the nanolayered PET is higher than that of the bulk material. The result is attributed to the diffusion of PC molecules into the PC layers and/or the interaction between the PET molecules and the interphases. In addition, it is further observed that the crystallization exotherm substantially broadens and shifts towards higher temperatures when the size of the layers is reduced up to $60 \mathrm{~nm}$. This result is associated to the heterogeneity of the system, with regions of different dynamics at a local level.

Real time SAXS studies during the initial stages of crystallization at $150{ }^{\circ} \mathrm{C}$ of nanolayered PET reveal that lamellae oriented with the basal surface parallel to the layer stacking (flat-on lamellae) appear. The orientation distribution broadens as crystallization proceeds up to a crystallization time of $\approx 20 \mathrm{~min}$. This broadening could be associated to new lamellae with slightly inclined surfaces that are inserted between the first developed crystals. Analysis of the SAXS profiles reveals that nanolayered PET exhibits larger long period and amorphous layer thickness values for the first stages of the crystallization process than that of the PET control material. Results suggest that not only lamellar insertion but also some lamellar thickening takes place during crystallization. Finite size effects are found to delay the crystallization process. However, the final lamellar structure is similar for both, nanolayered and bulk material.

Room temperature SAXS measurements reveal that the preferential orientation of the lamellae is retained upon cooling. Moreover, an enhanced orientation of the flat-on lamellar crystals is observed at room temperature with respect to that determined at the end of the isothermal crystallization at $150{ }^{\circ} \mathrm{C}$. In addition, thinner crystals separated by larger amorphous regions are generated during the cooling process.

Room temperature WAXS measurements of the crystallized multilayered materials reveal uniaxial symmetry and indicate that edge-on lamellae are also generated upon crystallization. The simulation of uniaxially oriented patterns reveals that the $c$-axis of the edge-on lamellae lies parallel to the layer surfaces. It is proposed that two lamellar populations develop: edge-on lamellae appear close to the interphases while flat-on lamellae are preferentially located in the layer core.

\section{Acknowledgements}

The authors wish to thank the $\mathrm{MCI}$ (Ministerio de Ciencia e Innovación), Spain, for funding the research reported under the grant FIS2007-60534. This research was also funded by the NSF Center for Layered Polymeric Systems (grant DMR-0423914). We are also indebted to the Department of Crystallography (Instituto Química-Física "Rocasolano", CSIC) for providing the facilities to carry out the wide-angle X-ray diffraction experiments. Thanks are also due to the Group of Properties of Polymeric Materials (ETSI Aeronáuticos, UPM) for providing the facilities to carry out some of the DSC measurements. Synchrotron radiation experiments at the Spanish operated BM16-CRG beamline (ESRF, Grenoble) were financially supported by the Ministerio de Ciencia y Tecnología, Spain. Finally, the authors are also indebted to Dr. Pawel Sikorski for his valuable help with the Clearer2 program.

\section{References}

[1] Zhu L, Cheng SZD, Calhoun BH, Ge Q, Quirk RP, Thomas EL, et al. J Am Chem Soc 2000;122(25):5957-67.

[2] Darling SB. Prog Polym Sci 2007;32:1152-204.

[3] Matyjaszewski K. Prog Polym Sci 2005;30:858-75.

[4] Knoll A, Magerle R, Krausch G. J Chem Phys 2004:120(2):1105-16.

[5] Thurn-Albrecht T, Schotter J, Kästle GA, Emley N, Shibauchi T, Krusin-Elbaum T, et al. Science 2000;290:2126-9.

[6] Underhill RS, Liu GJ. Chem Mater 2000;12(8):2082-91.

[7] Gao C, Yan D. Prog Polym Sci 2004;29:183-275.

[8] Tessler N, Medvedev V, Kazes M, Kan S, Banin U Science 2002:295:1506-8.

[9] Liu RYF, Jin Y, Hiltner A, Baer E. Macromol Rapid Commun 2003;24:943-8.

[10] Sinha Ray S, Okamoto M. Prog Polym Sci 2003;28:1539-41.

[11] Sun YM, Steinhart M, Zschech D, Adhikari R, Michler GH, Gosele U. Macromol Rapid Commun 2005;26:369-75.

[12] Theron A, Zussman E, Yarin AL. Nanotechnology 2001;12(3):384-90.

[13] Forrest JA, Danoki-Veress K. Adv Colloid Interface Sci 2001;94:167-96.

[14] Alcoutlabi M, McKenna GB. J Phys Condens Matter 2005;17:R461-524.

[15] Forrest JA, Dalnoki-Veress K, Stevens JR. Phys Rev Lett 1996;77:2002-5.

[16] Roth CB, Dutcher JR. Eur Phys J E 2003;12:S103-7.

[17] Serghei A, Tress M, Kremer F. J Chem Phys 2009;131:154904.

[18] Porter CE, Blum FD. Macromolecules 2000;33(19):7016-20.

[19] Roth CB, Torkelson JM. Macromolecules 2007;40:3328-36.

[20] Liu RYF, Bernal-Lara TE, Hiltner A, Baer E. Macromolecules 2005;38:4819-27.

[21] Liu RYF, Bernal-Lara TE, Hiltner A, Baer E. Macromolecules 2004;37:6972-9.

[22] Wang H, Keum JK, Hiltner A, Baer E, Freeman B, Rozanski A, et al. Science 2009;323:757-60.

[23] Schönherr H, Frank CW. Macromolecules 2003;36:1188-98.

[24] Despotopoulou MM, Frank CW, Miller RD, Rabolt JF. Macromolecules 1996;29:5797-804.

[25] Baltá-Calleja FJ, Ania F, Puente-Orench I, Baer E, Hiltner A, Bernal T, et al. Prog Colloid Polym Sci 2005;130:140-8.

[26] Puente-Orench I, Stribeck N, Ania F, Baer E, Hiltner A, Baltá-Calleja FJ. Polymer 2009;50:2680-7.

[27] Bernal-Lara TE, Liu RYF, Hiltner A, Baer E. Polymer 2005:46:3043-55.

[28] Jin Y, Rogunova M, Hiltner A, Baer E, Nowacki R, Galeski A, et al. J Polym Sci B Polym Phys 2004;42:3380-96. 
[29] Wang Y, Chan C-M, Ng K-M. Macromolecules 2008;41:2548-53.

[30] Ma Y, Hu W, Reiter G. Macromolecules 2006;39:5159-64.

[31] Karim Oultache A, Kong X, Pellerin C, Brisson J, Pézolet M, Prud'homme RE. Polymer 2001;42:9051-8.

[32] Hammersley AP, Svensson SO, Thomson A. Nucl Instrum Meth A 1994;346:312-21.

[33] Stribeck N. X-ray scattering of soft matter. Berlin: Springer-Verlag; 2007.

[34] Murthy NS, Wang Z-G, Hsiao BS. Macromolecules 1999;32:5594-9.

[35] Wang Z-G, Hsiao BS, Murthy NS. J Appl Crystallogr 2000;33:690-4.

[36] Makin OS, Sikorski P, Serpell LC. J Appl Crystallogr 2007;40:966-72.

[37] Forrest JA, Dalnoki-Veress K, Dutcher JR. Phys Rev E 1997;56(5):5705-16.

[38] Fukao J, Miyamoto Y. Phys Rev E 2000;61:1743-54.

[39] Hall DB, Hooker JC, Torkelson JM. Macromolecules 1997;30:667-9.

[40] Gilmer JW, Wiswe D, Zachmann HG, Kugler J, Fischer EW. Polymer 1986;27:1391-5.
[41] Ellis A, Gordon D, King S, Jenkins M. Phys B Condens Matter 2006;385-386:514-6.

[42] Bark M, Zachmann HG. Acta Polymerica 1993:44:259-65.

[43] Jonas AM, Russell TP, Yoon DY. Colloid Polym Sci 1994;272:1344-51.

[44] Wang Z-G, Hsiao BS, Sauer BB, Kampert WG. Polymer 1999;40:4615-27.

[45] Lee B, Shin TJ, Lee SW, Yoon J, Kim J, Youn HS, et al. Polymer 2003;44:2509-18.

[46] Haubruge HG, Jonas AM, Legras R. Macromolecules 2004;37:126-34.

[47] Asano T, Baltá-Calleja FJ, Flores A, Tanigaki M, Mina F, Sawatari C, et al. Polymer 1999;40:6475-84.

[48] Göschel U, Deutscher K, Abetz V. Polymer 1996;37:1-6.

[49] Sakai Y, Imai M, Kaji K, Tsuji M. Macromolecules 1996;29:8830-4.

[50] Yang H, Bhimaraj P, Yang L, Siegel RW, Schadler L. J Polym Sci B Polym Phys 2007;45:747-57.

[51] Daubeny RD, Bunn CW, Brown CJ. Proc R Soc A 1954;226:531-42. 